\title{
Динамическая генерация спин-волнового тока в гибридных структурах
}

\author{
(С) И.И. Ляпилин ${ }^{+*}$, М.С. Окороков ${ }^{+}$ \\ + Институт фризики металлов Уральского отделения Российской академии наук, \\ 620990 Екатеринбург, Россия \\ * Уральский федеральный университет, \\ 620002 Екатеринбург, Россия \\ E-mail: Okorokovmike@gmail.com
}

(Получена 27 апреля 2016 г. Принята к печати 10 мая 2016 г.)

Методом неравновесного статистического оператора исследован спиновый транспорт поперек интерфейса в гибридной структуре полупроводник/ферромагнитный диэлектрик в условиях спинового эффекта Зеебека. Рассмотрено приближение эффективных параметров, когда каждая из рассматриваемых подсистем (электроны проводимости, магноны, фононы) характеризуется своей эффективной температурой. Рассмотрено влияние резонансного (электродипольного) возбуждения спиновой подсистемы электронов проводимости на возбуждение спин-волнового тока в диэлектрике. Построены макроскопические уравнения, описывающие спин-волновой ток, обусловленный как резонансным возбуждением спиновой системы электронов проводимости, так и наличием неоднородного температурного поля в ферромагнитном диэлектрике, учитывающие как резонансно-диффузионный характер распространения магнонов, так и процессы их релаксации. Показано, что в рассматриваемых условиях возбуждение спин-волнового тока также имеет резонансный характер.

\section{1. Введение}

Изучение спинового эффекта Зеебека (СЭЗ) в структурах металл/магнитный изолятор (nomal metal/ferromagnet insulator, $\mathrm{N} / \mathrm{F}) \mathrm{LaY}_{2} \mathrm{Fe}_{5} \mathrm{O}_{12}[1]$ показало, что в отличие от проводящих кристаллов, где перенос спинового углового момента обусловлен зонными носителями заряда, в непроводящих магнитных материалах реализация СЭ3 связана с возбуждением системы локализованных спинов. Перенос углового момента при СЭЗ обусловлен спин-волновым током (спиновой волной), основу которого составляют возбуждения подсистемы локализованных спинов (магноны). В силу малого затухания спиновых волн спин-волновой ток распространяется на значительно бо́льшие расстояния, чем электронный спиновый ток, что позволяет надеяться на возможные практические применения эффекта [2,3]. Экспериментально спин-волновой ток исследован в работе [4].

Реализация СЭ3 в непроводящем магнетике гибридной структуры металл/ферромагнитный диэлектрик требует создания магнонной аккумуляции в непроводящем магнетике. В экспериментах по СЭЗ такое неравновесное распределение магнонов создается вследствие неупругого рассеяния спин-поляризованных электронов на локализованных моментах, расположенных на интерфейсе $(\mathrm{N} / \mathrm{F})$, сопровождающееся рождением (или аннигиляцией) магнонов. Доминирование процессов рассеяния с рождением магнонов обусловлено наличием спиновой аккумуляции (спиновой поляризации) электронов проводимости металла. Поляризация электронов в немагнитном материале достигается вследствие спинового эффекта Холла [5], величина которого зависит от величины спин-орбитального взаимодействия в системе электронов проводимости. Именно поэтому для получения достаточно заметной электронной спиновой аккумуляции применяется платина - металл, в котором спин-орбитальное взаимодействие достаточно велико.

Представляет интерес рассмотреть и иные методы реализации спиновой аккумуляции, необходимые для наблюдения СЭ3, среди которых следует выделить резонансные. Заметим, что в аналогичных гибридных структурах был реализован эффект электронной спиновой накачки - создание электронного спинового тока посредством возмущения магнитной подсистемы переменным магнитным полем в условиях ферромагнитного резонанса. Применение данного метода позволило получить электронный спиновый ток без переноса спин-поляризованных носителей заряда через интерфейс, избежав тем самым проблемы несоответствия проводимостей (mismath problem) [6-8], препятствующей получению высоких значений спиновой поляризации путем обычной спиновой инжекции. Данный метод неприменим в случае СЭЗ, поскольку здесь требуется обратное воздействие - передача углового момента от электронной системы в систему локализованных магнитных моментов. Однако спин-орбитальное взаимодействие позволяет в принципе и реализацию другого „резонансного“ сценария.

Связывая кинетическую (трансляционную) и спиновую подсистемы электронов проводимости, спинорбитальное взаимодействие (СОВ) можно рассматривать как канал, который позволяет воздействовать на одну из подсистем через другую (например, на спиновую подсистему электронов проводимости через кинетическую подсистему и наоборот). Такое „зацепление“ трансляционного и спинового движений делает невозможным разделение квантовых переходов на чисто конфигурационные (орбитальные) и чисто спиновые. 
Можно говорить лишь о преимущественно конфигурационных и преимущественно спиновых переходах. Это существенно изменяет условия возбуждения различных переходов, а именно становится возможным возбуждение спиновых переходов электрической компонентой электромагнитного поля, а орбитальных переходов магнитной компонентой. СОВ приводит к возможности резонансных переходов электронов на частотах, представляющих собой линейные комбинации циклотронной и зеемановской частот, причем такого рода переходы возможны в пучности как электрического, так и магнитного полей. Резонанс такого типа известен как комбинированный резонанс Рашбы [9-12] и наблюдался в ряде соединений. Существенно, что мощность, поглощаемая электронами при насыщении комбинированного резонанса (КР) в пучности электрического поля, на несколько порядков больше мощности, поглощаемой при насыщении парамагнитного резонанса (ПР).

Если исходить из вышеизложенного, представляет интерес изучить влияние резонансного возбуждения электронов в системе полупроводник/магнитный диэлектрик на генерацию спин-волнового тока в непроводящем ферромагнетике. Для краткости будем полагать, что в полупроводнике реализован электродипольный резонанс, а спиновая релаксация электронов обусловлена обменным взаимодействием их с локализованными спинами, расположенными на интерфейсе. В качестве дополнительного механизма рассеяния магнонов рассмотрим магнонфононное взаимодействие.

\section{2. Гамильтониан и неравновесный статистический оператор}

Решение поставленной задачи сводится к построению и решению системы макроскопических уравнений для спиновых подсистем: электронов проводимости $\left\langle\dot{s}^{z}\right\rangle^{t}$ и локализованных спинов диэлектрика $\left\langle\dot{S}^{z}\right\rangle^{t}$. Здесь

$$
\dot{A}=(i \hbar)^{-1}[A, H], \quad\langle A\rangle^{t}=\operatorname{Sp}(\rho(t) A),
$$

$H$ - гамильтониан рассматриваемой системы,

$$
H=H_{S C}+H_{F I}+H_{L}+H_{F} .
$$

Система электронов проводимости описывается гамильтонианом $H_{S C}$ :

$$
\begin{gathered}
H_{S C}=\int d \mathbf{x} H_{e}(\mathbf{x}), \\
H_{e}(\mathbf{x})=\sum_{j}\left\{H_{e, j}, \delta\left(\mathbf{x}-\mathbf{x}_{j}\right)\right\}, \\
H_{e, j}=H_{k, j}+H_{s, j}+H_{k s, j}, \\
H_{k}=\sum_{j} \frac{p_{j}^{2}}{2 m}, \quad H_{s}=-\hbar \omega_{s} \sum_{j} s_{j}^{z} .
\end{gathered}
$$

$H_{k s}$ описывает взаимодействие кинетических и спиновых степеней свободы электронов, которое в общем виде можно записать как

$$
H_{k s}=\sum_{j} f\left(\mathbf{p}_{j}\right) S_{j}=R \sum_{j} p_{j}^{\alpha_{1}} p_{j}^{\alpha_{2}} \ldots p_{j}^{\alpha_{s}} S_{j}^{\beta} .
$$

Здесь $R$ - константа, зависящая от силы спин-орбитального взаимодейстия, а $f\left(\mathbf{p}_{j}\right)$ - псевдовектор, компоненты которого представляют формулу порядка $s$ от компонент кинетического импульса $p_{j}^{\alpha} ; s_{j}^{z}$ и $p_{j}^{\gamma}-$ компоненты операторов спина и кинетического импульса $j$ го электрона соответственно; $\omega_{s}=g_{s} \mu_{0} H / \hbar-$ частота зеемановской прецессии свободных электронов во внешнем магнитном поле, которое направлено вдоль оси $z$. $\left(g_{s}, \mu_{0}-\right.$ эффективный фактор спектроскопического расщепления электронов и магнетон Бора соответственно); $\{A, B\}=(A B+B A) / 2$.

Теоретическое описание КР основано на малости взаимодействия $H_{k s}[9,11]$, что позволяет построить „новый“ эффективный гамильтониан путем канонического преобразования исходного гамильтониана. Результат преобразования - устранение взаимодействия спиновых и кинетических степеней свободы электронов в линейном по $H_{k s}$ приближении. При этом перенормируется и взаимодействие электронной системы с электромагнитным полем, определяющим резонансное поглощение энергии. Будем полагать, что каноническое преобразование гамильтониана выполнено, и перенормированное взаимодействие с переменным электрическим полем $\mathbf{E}(t)$ имеет вид $[12]$

$$
\begin{aligned}
& \bar{H}_{e f}(t)=[\mathbf{r}, T(p)] e \mathbf{E}(t), \\
& T(p)=R \sum \frac{T^{\alpha_{1}, \ldots \alpha_{s} ; \beta}}{\hbar \Omega_{\alpha_{1}, \ldots \alpha_{s} ; \beta}},
\end{aligned}
$$

где $T(p)$ - оператор канонического преобразования, a $\Omega_{\alpha_{1}, \ldots \alpha_{s} ; \beta}-$ некоторая линейная комбинация циклотронной, $\omega_{0}$, и зеемановской, $\omega_{s}$, частот электронов, зависящая от конкретной структуры оператора $T^{\alpha_{1}, \ldots \alpha_{s} ; \beta}$. Резонансу на зеемановской частоте соответствует оператор преобразования $T(p)$, линейный по импульсу.

Подсистема локализованных спинов диэлектрика описывается гамильтонианом $H_{F I} . H_{L}-$ гамильтониан решетки; $H_{F}$ описывает взаимодействие электронов проводимости с переменным электрическим полем; $\rho(t)-$ неравновесный статистический оператор (НСО) (или матрица плотности).

Описание неравновесного состояния рассматриваемой системы состоит в определении средних энергий подсистем $(s),(m)$ или термодинамически сопряженных с ними обратных эффективных температур $\beta_{s}, \beta_{m}$ и соответствует случаю, когда скорости процессов установления внутреннего равновесия каждой из подсистем велики по сравнению со скоростью обмена энергией между подсистемами. Аналогичный подход использовался нами при описании СЭЗ в гибридных структурах [13]. 
Заметим, что при реализации комбинированных переходов следует ожидать отклонения от равновесия как кинетической, так и спиновой подсистем электронов проводимости. Однако в случае резонанса на спиновой частоте поглощение энергии внешнего переменного поля связано только со спиновой подсистемой электронов проводимости. При этом можно полагать, что кинетическая подсистема электронов проводимости слабо отклоняется от равновесного состояния. Поэтому в условиях насыщения комбинированного (спинового) резонанса в электронной подсистеме достаточно рассмотреть эволюцию только двух спиновых подсистем: спиновой проводимости и подсистемы локализованных спинов. В приближении эффективных параметров спиновую подсистему электронов будем характеризовать температурой $T_{s}$, а подсистему локализованных спинов ферромагнитного диэлектрика (индекс FI) температурой $T_{m}$. Фононной подсистеме соответствует равновесная температура $T$.

Гамильтониан магнитной подсистемы $H_{F I}$ запишем в виде

$$
H_{F I}=\int d \mathbf{x}\left[H_{m}(\mathbf{x})+H_{s m}(\mathbf{x})\right] .
$$

Здесь $H_{m}(\mathbf{x})$ - оператор плотности энергии магнитной подсистемы, представляющий собой сумму обменных (по ближайшим соседям), $H_{S S}$, и зеемановской энергии, $H_{S}$ :

$$
H_{S S}=-J \sum_{j \delta} S_{j} S_{j+\delta}, \quad H_{S}=-\hbar \omega_{m} \sum_{j} S_{j}^{z},
$$

где $J$ - обменный интеграл, $\omega_{m}=g_{m} \mu_{0} H / \hbar ; H_{s m}(\mathbf{x})-$ плотность энергии обменного взаимодействия с электронами проводимости на интерфейсе,

$$
H_{s m}=-J_{0} \sum_{j} \int d \mathbf{x} s(\mathbf{x}) S\left(\mathbf{R}_{j}\right) \delta\left(\mathbf{x}-\mathbf{R}_{j}\right),
$$

$J_{0}$ - обменный интеграл, $S\left(\mathbf{R}_{j}\right)$ - оператор локализованного спина с координатой $\mathbf{R}_{j}$ на интерфейсе.

Гамильтониан решетки есть

$$
H_{L}=\int d \mathbf{x}\left[H_{p}(\mathbf{x})+H_{p m}(\mathbf{x})\right]
$$

где $H_{p}(\mathbf{x})$ - оператор плотности энергии фононной подсистемы, $H_{p m}(\mathbf{x})$ - оператор плотности энергии взаимодействия локализованных спинов с фононами.

Неравновесный статистический оператор ( $\mathrm{HCO}) \rho(t)$ в линейном приближении по отклонению от равновесия может быть записан в виде [13-15]

$$
\rho(t)=\rho_{q}(t)-\int_{-\infty}^{0} d t_{1} e^{\epsilon t_{1}} \int_{0}^{1} d \tau \rho_{0}^{\tau} \dot{S}\left(t+t_{1}, t_{1}\right) \rho_{0}^{-\tau} \rho_{0} .
$$

Здесь $\rho_{q}(t)=\exp \{-S(t)\}-$ квазиравновесный статистический оператор, $\dot{S}(t)$ - оператор производства энтропии,

$$
\dot{S}(t)=\delta \dot{S}(t)=\frac{\partial S(t)}{\partial t}+\frac{1}{i \hbar}[S(t), H] .
$$

Задача построения оператора $\rho(t)$ сводится к нахождению оператора $\dot{S}(t)$. В терминах средних значений плотностей оператор энтропии, соответствующий неравновесному состоянию системы, запишем в виде

$$
\begin{aligned}
S(t) & =\Phi(t)+\int d \mathbf{x}\left\{\beta\left[H_{k}(\mathbf{x})-\mu(\mathbf{x}, t) N(\mathbf{x}, t)\right]\right. \\
& +\beta_{s}(\mathbf{x}, t)\left[H_{s}(\mathbf{x}, t)+(1 / 2) H_{s m}(\mathbf{x}, t)\right] \\
& +\beta_{m}(\mathbf{x}, t)\left[H_{m}(\mathbf{x}, t)+(1 / 2) H_{s m}(\mathbf{x}, t)+(1 / 2) H_{p m}(\mathbf{x}, t)\right] \\
& \left.+\beta\left[H_{p}(\mathbf{x}, t)+(1 / 2) H_{p m}(\mathbf{x}, t)\right]\right\}=S_{0}+\delta S(t),
\end{aligned}
$$

где $\Phi(t)$ - функционал Масье-Планка; $\beta_{i}(\mathbf{x}, t), i=s$, $m$ - локально-равновесные значения обратных температур подсистем $(s),(m)$ соответственно; $\mu$ - локально-равновесное значение химического потенциала электронов; $N(\mathbf{x})=\sum_{i} \delta\left(\mathbf{x}-\mathbf{x}_{i}\right)$ - оператор плотности числа электронов; $\beta^{-1}=T-$ равновесная температура системы; $S_{0}$ - энтропия равновесной системы с гамильтонианом $H$,

$$
S_{0}=\Phi_{0}+\beta\left(H_{e}-\mu N\right)+\beta\left(H_{m}+H_{p}+H_{s m}+H_{m p}\right) .
$$

Оператор $\delta S(t)=\int d \mathbf{x} \delta S(\mathbf{x}, t)$ описывает отклонение системы от равновесного состояния. Заметим, что при записи оператора энтропии мы пренебрегли как величинами второго порядка малости перенормированными энергиями взаимодействия $\left(\left[T(\mathbf{p}), H_{s m}\right],\left[T(\mathbf{p}), H_{p m}\right]\right.$ и т. Д.),

$$
\Delta A=A-\langle A\rangle_{0}, \quad\langle\ldots\rangle_{0}=\operatorname{Sp}\left(\ldots \rho_{0}\right)
$$

\section{3. Микроскопические уравнения}

Операторные уравнения движения найдем, коммутируя базисные операторы $H_{s}(\mathbf{x}), N(\mathbf{x}), H_{m}(\mathbf{x})$ с гамильтонианом $H$. Имеем

$$
\begin{gathered}
\dot{N}(\mathbf{x})=-\nabla I_{N}(\mathbf{x}), \\
\dot{H}_{s}(\mathbf{x})=-\nabla I_{H_{s}}(\mathbf{x})+(1 / 2) \dot{H}_{s(s m)}(\mathbf{x})+\dot{H}_{s(e F)}(\mathbf{x}) .
\end{gathered}
$$

Здесь $I_{N}(\mathbf{x})=(1 / m) \sum_{j}\left\{p_{j}, \delta\left(\mathbf{x}-\mathbf{x}_{j}\right)\right\}, I_{H_{s}}(\mathbf{x})=-\hbar \omega_{s}(1 / m)$ $\times \sum_{j} s_{j}^{z}\left\{p_{j}, \delta\left(\mathbf{x}-\mathbf{x}_{j}^{\alpha}\right)\right\}-$ плотности потоков частиц и зеемановской энергии; $\dot{H}_{s(s m)}(\mathbf{x})-$ скорость изменения локальной энергии электронов за счет взаимодействия $H_{s m}(\mathbf{x}), \dot{H}_{s(e F)}(\mathbf{x})-$ изменение энергии электронов за счет взаимодействия с электрическим полем, $\dot{A}_{\lambda(\lambda \gamma)}(\mathbf{x})=(i \hbar)^{-1}\left[A_{\lambda}(\mathbf{x}), H_{\lambda \gamma}\right]$.

Преобразуем гамильтониан подсистемы локализованных спинов к спин-волновым (магнонным) переменным, 
переходя к магнонным операторам рождения, $b_{\mathbf{k}}^{+}$, и аннигиляции, $b_{\mathbf{k}}[16]$. Рассматривая магнонный газ как свободный, имеем

$$
H_{m}=\sum_{k} \varepsilon(k) b_{k}^{+} b_{k}, \quad \varepsilon(k)=\frac{\hbar^{2} k^{2}}{2 m^{*}} .
$$

Это выражение можно трактовать как сумму энергий квазичастиц-магнонов, обладающих квазиимпульсом $(\hbar k)$ со своей эффективной массой $m^{*}$ и магнитным моментом [17]. Уравнение движения для магнитной подсистемы можно записать в виде

$$
\dot{H}_{m}(\mathbf{x})=-\nabla I_{H_{m}}(\mathbf{x})+(1 / 2) \dot{H}_{m(s m)}(\mathbf{x})+(1 / 2) \dot{H}_{m(p m)}(\mathbf{x}) .
$$

Здесь $I_{H_{m}}(\mathbf{x})=-\hbar \omega_{m} I_{S^{z}}(\mathbf{x})-$ плотность потока магнонной энергии. Остальные слагаемые в правой части уравнения определяют процессы рассеяния магнонов на интерфейсе и фононах. Неупругая часть обменного взаимодействия, записанная через операторы рождения и аннигиляции электронов и магнонов, имеет вид

$$
H_{s m}=-J^{*} \sum_{k, k^{\prime}, q}\left\{b_{q}^{+} a_{k \uparrow}^{+} a_{k^{\prime} \downarrow}+b_{q} a_{k^{\prime} \downarrow}^{+} a_{k \uparrow}\right\} \delta_{\mathbf{k}^{\prime}, \mathbf{k}+\mathbf{q}},
$$

где $a_{k \alpha}^{+}\left(a_{k \alpha}\right)$ операторы рождения (аннигиляции) электронов с определенным значением спина $\alpha=\uparrow, \downarrow$.

Наконец, уравнение движения для решеточной подсистемы имеет вид

$$
\dot{H}_{p}(\mathbf{x})=-\nabla I_{H_{p}}(\mathbf{x})+(1 / 2) \dot{H}_{p(p m)}(\mathbf{x}) .
$$

Принимая во внимание явный вид уравнений движения для базисных операторов, запишем оператор производства энтропии в виде

$$
\begin{aligned}
& \dot{S}(t)=\Delta \int d \mathbf{x}\left\{-\beta \delta \mu(\mathbf{x}, t) \nabla I_{N}(\mathbf{x})\right. \\
& \quad+\beta \dot{H}_{s(e F)}(\mathbf{x}, t)+\delta \beta_{s}(\mathbf{x}, t)\left[-\nabla I_{H_{s}}(\mathbf{x})+\dot{H}_{s(s m)}(\mathbf{x})\right] \\
& \left.\quad+\delta \beta_{m}(\mathbf{x}, t)\left[-\nabla I_{H_{m}}(\mathbf{x})+\dot{H}_{m(s m)}(\mathbf{x})+\dot{H}_{m(m p)}(\mathbf{x})\right]\right\} .
\end{aligned}
$$

Интегрируя далее по частям слагаемые, содержащие дивергенции потоков, и отбрасывая поверхностные интегралы, получаем

$$
\begin{aligned}
& \dot{S}(t)=\Delta \int d \mathbf{x}\left\{I_{N}(\mathbf{x}) \beta \nabla \mu(\mathbf{x}, t)\right. \\
& \quad+I_{H_{s}}(\mathbf{x}) \nabla \beta_{s}(\mathbf{x}, t)+I_{H_{m}}(\mathbf{x}) \nabla \beta_{m}(\mathbf{x}, t) \\
& \quad+\beta \dot{H}_{s(e F)}(\mathbf{x}, t)+\delta \beta_{s}(\mathbf{x}, t)\left[(1 / 2) \dot{H}_{s(s m)}(\mathbf{x})\right] \\
& \left.\quad+\delta \beta_{m}(\mathbf{x}, t)(1 / 2)\left[\dot{H}_{m(s m)}(\mathbf{x})+(1 / 2) \dot{H}_{m(p m)}(\mathbf{x})\right]\right\} .
\end{aligned}
$$

Разлагая квазиравновесный оператор $\rho_{q}(t)=\exp \{-S(t)\}$ по степеням $\delta S(t)$, найдем линейную связь между откло- нениями термодинамических координат и термодинамических сил от их равновесных значений. Имеем

$$
\begin{aligned}
\delta\left\langle H_{s}(\mathbf{x})\right\rangle^{t}= & -\int d \mathbf{x}^{\prime}\left\{\delta \beta_{s}\left(\mathbf{x}^{\prime}, t\right)\left(H_{s}(\mathbf{x}), H_{s}\left(\mathbf{x}^{\prime}\right)\right)_{0}\right. \\
& \left.-\beta \delta \mu\left(\mathbf{x}^{\prime}, t\right)\left(H_{s}(\mathbf{x}), N\left(\mathbf{x}^{\prime}\right)\right)_{0}\right\} \\
\delta\langle N(\mathbf{x})\rangle^{t}= & -\int d \mathbf{x}^{\prime}\left\{\delta \beta_{s}\left(\mathbf{x}^{\prime}, t\right)\left(N(\mathbf{x}), H_{s}\left(\mathbf{x}^{\prime}\right)\right)_{0}\right. \\
& \left.-\beta \delta \mu\left(\mathbf{x}^{\prime}, t\right)\left(N(\mathbf{x}), N\left(\mathbf{x}^{\prime}\right)\right)_{0}\right\} \\
\delta\left\langle H_{m}(\mathbf{x})\right\rangle^{t}=- & \int d \mathbf{x}^{\prime} \delta \beta_{m}\left(\mathbf{x}^{\prime}, t\right)\left(H_{m}(\mathbf{x}), H_{m}\left(\mathbf{x}^{\prime}\right)\right)_{0},
\end{aligned}
$$

где

$$
\delta\langle A\rangle^{t}=\langle A\rangle^{t}-\langle A\rangle_{0}, \quad(A, B)_{0}=\int_{0}^{1} d \lambda \operatorname{Sp}\left\{A \rho_{0}^{\lambda} \Delta B \rho_{0}^{1-\lambda}\right\}
$$

Принимая во внимание, что $\langle N(\mathbf{x})\rangle^{t}=\langle N(\mathbf{x})\rangle_{0}$ и переходя к фурье-компонентам по пространственным координатам, получаем:

$$
\begin{gathered}
\beta \delta \mu(\mathbf{q}, t)=-\frac{\left(N(\mathbf{q}), H_{s}(-\mathbf{q})\right)_{0}}{(N(\mathbf{q}), N(-\mathbf{q}))_{0}} \delta \beta_{s}(\mathbf{q}, t), \\
\delta\left\langle H_{s}(\mathbf{q})\right\rangle^{t}=-\delta \beta_{s}(\mathbf{q}, t)\left(\hbar \omega_{s}\right)^{2} C_{z z}(\mathbf{q}),
\end{gathered}
$$

где

$$
\begin{gathered}
C_{z z}(\mathbf{q})=\left(s^{z}(\mathbf{q}), s^{z}(-\mathbf{q})\right)_{0}-\frac{\left(s^{z}(\mathbf{q}), N(-\mathbf{q})\right)_{0}\left(N(\mathbf{q}), s^{z}(-\mathbf{q})\right)_{0}}{(N(\mathbf{q}), N(-\mathbf{q}))_{0}} \\
\delta\left\langle H_{m}(\mathbf{q})\right\rangle^{t}=-\delta \beta_{m}(\mathbf{q}, t)\left(\hbar \omega_{m}\right)^{2}\left(S^{z}(\mathbf{q}), S^{z}(-\mathbf{q})\right)_{0} \\
\equiv-\delta \beta_{m}(\mathbf{q}, t)\left(\hbar \omega_{m}\right)^{2} C_{S S} .
\end{gathered}
$$

\section{4. Макроскопические уравнения}

Макроскопические уравнения для плотности спиновой намагниченности электронов проводимости $\delta m^{z}(\mathbf{x}, t)=g_{s} \mu_{0} \delta\left\langle s^{z}(\mathbf{x})\right\rangle^{t} \quad$ и $\quad$ локализованных спинов $\delta M^{z}(\mathbf{x}, t)=g_{m} \mu_{0} \delta\left\langle S^{z}(\mathbf{x})\right\rangle^{t}$ найдем, усреднив соответствующие микроскопические операторные уравнения с помощью $\mathrm{HCO}$ (7). Имеем

$$
\begin{aligned}
\frac{\partial}{\partial t} \delta m^{z}(\mathbf{x}, t)= & -\nabla\left\langle I_{H_{s}}(\mathbf{x}, t)\right\rangle^{t}+\left\langle\dot{H}_{s(s m)}(\mathbf{x}, t)\right\rangle^{t} \\
& +\left\langle\dot{H}_{s(e F)}(\mathbf{x}, t)\right\rangle^{t} \\
\frac{\partial}{\partial t} \delta M^{z}(\mathbf{x}, t)= & -\nabla\left\langle I_{H_{m}}(\mathbf{x}, t)\right\rangle^{t}+\left\langle\dot{H}_{m(s m)}(\mathbf{x}, t)\right\rangle^{t} \\
& +\left\langle\dot{H}_{m(p m)}(\mathbf{x}, t)\right\rangle^{t} .
\end{aligned}
$$

Уравнения (19) описывают изменение плотности спиновой намагниченности электронной и магнитной подсистем вследствие процессов диффузии (первые слагаемые 
в правых частях уравнений), релаксационных процессов вследствие обменного взаимодействия электронов с локализованными моментами на интерфейсе (слагаемое $\left.\left\langle\dot{H}_{i(s m)}(\mathbf{x}, t)\right\rangle^{t}, i=s, m\right)$ и поглощение энергии внешнего электрического поля спиновой подсистемой электронов проводимости $\left\langle\dot{H}_{s(e F)}(\mathbf{x}, t)\right\rangle^{t} \equiv Q_{s}(\mathbf{x}, t)$. Слагаемые $\left\langle\dot{H}_{m(p m)}(\mathbf{x}, t)\right\rangle^{t}$ определяют релаксацию магнонов на фононах. Выражения для этих коэффициентов следующие:

$$
\begin{aligned}
& \left\langle I_{H_{s}}^{\alpha}(\mathbf{x}, t)\right\rangle^{t}=\int d x^{\prime} \int_{-\infty}^{0} d t_{1} e^{\epsilon t_{1}}\left(I_{H_{s}}^{\alpha}(\mathbf{x}), I_{H_{s}}^{\lambda}\left(\mathbf{x}^{\prime}, t_{1}\right)\right)_{0} \\
& \times \nabla \beta_{s}^{\lambda}\left(\mathbf{x}^{\prime}, t+t_{1}\right)+\left(I_{H_{s}}^{\alpha}(\mathbf{x}), I_{N}^{\alpha}\left(\mathbf{x}^{\prime}, t_{1}\right)\right)_{0} \beta \nabla \mu^{\alpha}\left(\mathbf{x}^{\prime}, t+t_{1}\right) \\
& +\left(I_{H_{s}}^{\alpha}(\mathbf{x}), I_{H_{m}}^{\lambda}\left(\mathbf{x}^{\prime}, t_{1}\right)\right)_{0} \nabla \beta_{m}^{\lambda}\left(\mathbf{x}^{\prime}, t+t_{1}\right), \\
& \left\langle I_{H_{m}}^{\alpha}(\mathbf{x}, t)\right\rangle^{t}=\int d x^{\prime} \int_{-\infty}^{0} d t_{1} e^{\epsilon t_{1}}\left(I_{H_{m}}^{\alpha}(\mathbf{x}), I_{H_{m}}^{\lambda}\left(\mathbf{x}^{\prime}, t_{1}\right)\right)_{0} \\
& \times \nabla \beta_{m}^{\lambda}\left(\mathbf{x}^{\prime}, t+t_{1}\right)+\left(I_{H_{m}}^{\alpha}(\mathbf{x}), I_{H_{s}}^{\gamma}\left(\mathbf{x}^{\prime}, t_{1}\right)\right) \nabla \beta_{s}^{\gamma}\left(\mathbf{x}^{\prime}, t+t_{1}\right) \\
& +\left(I_{H_{m}}^{\alpha}(\mathbf{x}), I_{H_{p}}^{\lambda}\left(\mathbf{x}^{\prime}, t_{1}\right)\right)_{0} \nabla \beta_{m}^{\lambda}\left(\mathbf{x}^{\prime}, t+t_{1}\right) \\
& \left\langle\dot{H}_{i(j n)}(\mathbf{x}, t)\right\rangle^{t}=\int d x^{\prime} \int_{-\infty}^{0} d t_{1} e^{\epsilon t_{1}}\left(\dot{H}_{i(j n)}(\mathbf{x}), \dot{H}_{i(j n)}\left(\mathbf{x}^{\prime}, t_{1}\right)\right)_{0} \\
& \times \delta \beta_{j}\left(\mathbf{x}^{\prime}, t+t_{1}\right), \quad i, j=s, m, \quad n=m, p, \\
& Q(\mathbf{x}, t)=\beta \int d x^{\prime} \int_{-\infty}^{0} d t_{1} e^{\epsilon t_{1}}\left(\dot{H}_{s(e F)}(\mathbf{x}), \dot{H}_{s(e F)}\left(\mathbf{x}^{\prime}, t+t_{1}\right)\right)_{0} .
\end{aligned}
$$

Уравнения (19) и кинетические коэффициенты (20)-(23) решают задачу макроскопического описания неравновесных спиновых подсистем в терминах средних плотностей намагниченностей.

Рассматривая далее случай стационарного течения энергии между подсистемами, усредним уравнения по времени $t$ согласно правилу

$$
\overline{A(t)}=\epsilon \int_{-\infty}^{0} d t A(t) e^{\epsilon t}, \quad \epsilon \rightarrow+0 .
$$

При этом $\overline{\partial_{t} A(t)}=0, \overline{\delta \beta_{i}\left(\mathbf{x}, t+t_{1}\right)}=\delta \beta_{i}(\mathbf{x}), i=s, m, p$. $\mathrm{B}$ результате система уравнений $(23)$ примет вид

$$
\begin{gathered}
\delta \beta_{s m}(\mathbf{x}) L_{s(s m)}(\mathbf{x})+Q_{s}=0, \\
\delta \beta_{m s}(\mathbf{x}) L_{m(s m)}(\mathbf{x})+\delta \beta_{m p}(\mathbf{x}) L_{m(m p)}(\mathbf{x})=0 .
\end{gathered}
$$

Здесь $\delta \beta_{i k}=\beta_{i}-\beta_{k}$, а корреляционные функции $L_{i(j k)}(\mathbf{x})$ определены соотношениями

$$
\begin{gathered}
L_{i(j k)}(\mathbf{x})=\int_{-\infty}^{0} d t_{1} e^{\epsilon t_{1}} \int d \mathbf{x}^{\prime}\left(\dot{H}_{i(j k)}(\mathbf{x}), \dot{H}_{i(j k)}\left(\mathbf{x}^{\prime}, t_{1}\right)\right)_{0} \\
i, j=s, m, p
\end{gathered}
$$

Переходя в корреляционных функциях к фурье-представлению, положив

$$
\begin{gathered}
H_{s}(\mathbf{x})=\sum_{q} H_{s}(\mathbf{q}) e^{i \mathbf{q} \mathbf{x}}, \quad H_{s}(\mathbf{q})=\sum_{j} H_{s j} e^{-i \mathbf{q} \mathbf{x}_{j}}, \\
I(\mathbf{x})=\sum_{\mathbf{q}} I(\mathbf{q}) e^{i \mathbf{q} \mathbf{x}}, \quad I(\mathbf{q})=\sum_{j} p_{j} / m, e^{-i \mathbf{q} \mathbf{x}_{j}}, \ldots
\end{gathered}
$$

запишем выражение для средней мощности, поглощенной спиновой системой электронов проводимости в условиях КР $Q_{s}=\int d \mathbf{x} \overline{Q(\mathbf{x}, t)}$. Согласно [12], имеем

$$
\begin{aligned}
Q_{s}= & \beta \omega_{s}^{2} R^{2} \sum_{\mathbf{q}, \omega} \omega^{2}\left(s^{+}(\mathbf{q}), s^{-}(-\mathbf{q})\right)_{0}\left|E^{-}(\omega)\right|^{2} \\
& \times \frac{\Gamma(\mathbf{q}, \omega)}{\left(\omega-\omega_{s}\right)^{2}+\Gamma^{2}(\mathbf{q}, \omega)} .
\end{aligned}
$$

Здесь

$$
\Gamma(\mathbf{q}, \omega)=v(\mathbf{q}, \omega)+q^{\alpha} q^{\gamma} D_{\alpha, \gamma}^{ \pm}(\mathbf{q}, \omega),
$$

где $v(\mathbf{q}, \omega) \equiv v_{s}$ - известная формула для частоты релаксации поперечного спина электронов, определяющая ширину линии парамагнитного резонанса [18],

$$
\begin{aligned}
& v(\mathbf{q}, \omega)=\left(s^{+}(\mathbf{q}), s^{-}(-\mathbf{q})\right)_{0}^{-1} \\
& \times \operatorname{Re} \int_{-\infty}^{0} d t_{1} e^{(\epsilon-i \omega) t_{1}}\left(\dot{s}_{(e m)}^{+}(\mathbf{q}), \dot{s}_{(e m)}^{-}\left(-\mathbf{q}, t_{1}\right)\right)_{0},
\end{aligned}
$$

а $D_{\alpha, \gamma}^{ \pm}(\mathbf{q}, \omega)$ - тензор диффузии поперечных компонент спиновой намагниченности,

$$
\begin{aligned}
D_{\alpha, \gamma}^{ \pm}(\mathbf{q}, \omega) & =\left(s^{+}(\mathbf{q}), s^{-}(-\mathbf{q})\right)_{0}^{-1} \\
\times & \operatorname{Re} \int_{-\infty}^{0} d t_{1} e^{(\epsilon-i \omega) t_{1}}\left(I_{s^{+}}^{\alpha}(\mathbf{q}), I_{s^{-}}^{\gamma}\left(-\mathbf{q}, t_{1}\right)\right)_{0} .
\end{aligned}
$$

Выражения для кинетических коэффициентов $v(\mathbf{q}, \omega)$, $D_{\alpha, \gamma}^{ \pm}(\mathbf{q}, \omega)$ учитывают временну́ю и пространственную дисперсию, пригодны в области как квантующих, так и классических магнитных полей. Такого рода выражения для поглощенной мощности в однородном случае получались ранее в работах $[12,18]$.

Рассмотрим теперь систему уравнений (23) в стационарном случае. Имеем

$$
\begin{gathered}
Q_{s}(\mathbf{q}, \omega)+\left(\beta_{s}-\beta\right) L_{s(s m)}(\mathbf{q}, \omega)=0, \\
-q^{\alpha} q^{\gamma} D_{\alpha, \gamma}^{z z}(\mathbf{q}, \omega)+\left(\beta_{m}-\beta_{s}\right) L_{m(s m)}(\mathbf{q}, \omega) \\
+\left(\beta_{m}-\beta\right) L_{m(m p)}(\mathbf{q}, \omega)=0, \\
D_{\alpha, \gamma}^{z z}(q, \omega)=\left(S^{z}(q), S^{z}(-q)\right)_{0}^{-1} \\
\times \operatorname{Re} \int_{-\infty}^{0} d t_{1} e^{(\epsilon-i \omega) t_{1}}\left(I_{S^{z}}^{\alpha}(q), I_{S^{z}}^{\gamma}\left(-q, t_{1}\right)\right)_{0} .
\end{gathered}
$$


Из (28) следует, что спин-волновой ток в магнитном диэлектрике, который характеризуется отклонением намагниченности от равновесия, в однородном случае определяется выражением

$$
\begin{aligned}
\delta M^{z}(\mathbf{q}, \omega)= & -\beta\left(\frac{R \omega_{m}}{\omega_{s}}\right)^{2} \sum_{\omega} \omega^{2}\left(s^{+}, s^{-}\right)_{0}|E(\omega)|^{2} \\
& \times \frac{v_{s}}{\left(\omega-\omega_{s}\right)^{2}+v_{s}^{2}} \frac{1}{L_{m(s m)}+L_{m(m p)}}
\end{aligned}
$$

и резонансным образом зависит от частоты внешнего поля. Полагая, что основным механизмом рассеяния магнонов является релаксация их на фононах в условиях насыщения комбинированного резонанса, имеем

$$
\delta M^{z}(\mathbf{q}, \omega)=-\beta \frac{R^{2}\left(s^{+}, s^{-}\right)_{0}}{\left(S^{z}, S^{z}\right)_{0} v_{s} v_{m(m p)}}\left|E\left(\omega_{s}\right)\right|^{2},
$$

где $v_{m(m p)}$ - частота релаксации магнонов на фононах, определяемая выражением

$$
\begin{aligned}
v_{m(m p)}(\mathbf{q})= & \left(S^{z}(\mathbf{q}), S^{z}(-\mathbf{q})\right)_{0}^{-1} \\
& \times \int_{-\infty}^{0} d t_{1} e^{\epsilon t_{1}}\left(\dot{S}_{(m p)}^{z}(\mathbf{q}), \dot{S}_{(m p)}^{z}\left(-\mathbf{q}, t_{1}\right)\right)_{0} .
\end{aligned}
$$

Корреляционные функции $L_{i(j k)}$, описывающие релаксационные процессы при различных механизмах рассеяния, вычислялись в работах $[12,18]$.

\section{5. Заключение}

Методом неравновесного статистического оператора исследован спиновый транспорт поперек интерфейса в гибридной структуре полупроводник/ферромагнитный диэлектрик в условиях спинового эффекта Зеебека. Рассмотрено приближение эффективных параметров, когда каждая из рассматриваемых подсистем (электроны проводимости, магноны, фононы) характеризуется своей эффективной температурой.

Рассмотрено влияние резонансного (электродипольного) возбуждения спиновой подсистемы электронов проводимости на возбуждение спин-волнового тока в диэлектрике. Построены макроскопические уравнения, описывающие спин-волновой ток, обусловленный как резонансным возбуждением спиновой системы электронов проводимости, так и наличием неоднородного температурного поля в ферромагнитном диэлектрике, учитывающие как резонансно-диффузионный характер распространения магнонов, так и процессы их релаксации. Показано что в рассматриваемых условиях возбуждение спин-волнового тока также имеет резонансный характер.

Работа выполнена по теме „Спин“ 0120463330, при поддержке Министерства образования Российской Федерации (грант № 14.Z50.31.0025) и RFBR (грант 16-0200044) и проекта № 15-17-2-17.

\section{Список литературы}

[1] K. Uchida, T. Nonaka, T. Ota, E. Saitoh. Appl. Phys. Lett., 97, 262504 (2010).

[2] S. Takahashi, E. Saitoh, S. Maekawa. J. Phys: Conf. Ser., 200, 062030 (2010).

[3] F.L. Bakker, A. Schlachter, J.-P. Adam, B.H. van Wees. Phys. Rev. Lett., 105, 136601 (2010).

[4] Y. Kajiwara, K. Harii, S. Takahashi, J. Ohe, K. Uchida, M. Mizuguchi, H. Umezawa, H. Kawai, K. Ando, K. Takanashi, S. Maekawa, E. Saitoh. Nature, 464, 262 (2010).

[5] S.O. Valenzuela, M. Tinkham. Nature, 442, 176 (2006).

[6] H.J. Zhu, M. Ramsteiner, H. Kostial, M. Wassermeier, H.-P. Schonherr, K.H. Ploog. Phys. Rev. Lett., 87, 016601 (1996).

[7] B.T. Jonker, G. Kioseoglou, A.T. Hanbicki, C.H. Li, P.E. Thompson. Nature Phys., 3, 542 (2007).

[8] X. Lou, C. Adelmann, M. Furis, S.A. Crooker, C.J. Palmstrom, P.A. Growell. Phys. Rev. Lett., 96, 176603 (2006).

[9] E.I. Rashba. Sov. Phys. Usp., 84, 557 (1964).

[10] A.B. Roitsin. Sov. Phys. Usp., 14, 766 (1972).

[11] V.V. Kalashnikov. Theor. Mathem. Physics, 18, 76 (1974).

[12] V.V. Kalashnikov, I.I. Lyapilin. Theor. Mathem. Physics, 18, 194 (1974).

[13] I.I. Lyapilin, M.S. Okorokov, V.V. Ustinov. Phys. Rev. B, 91, 195309 (2015).

[14] Д.Н. Зубарев. Неравновесная статистическая термодинамика (М., Наука, 1971).

[15] D.N. Zubarev, V.V. Kalashnikov. Theor. Mathem. Physics, 1, 137 (1969).

[16] R.M. White. Quantum theory of magnetism (McGraw-Hill Book Company, 1970).

[17] Ферромагнитный резонанс. Сб. ст. под ред. С.В. Вонсовского (М., 1961).

[18] H.M. Bikkin, V.P. Kalashnikov. Theor. Mathem. Physics, 7, 79 (1971).

Редактор Л.В. Шаронова

\section{Dynamical generation of spin-wave currents into hybrid structures}

\section{I.I. Lyapilin ${ }^{+*}$, M.S. Okorokov ${ }^{+}$}

+ Institute of Metal Physics,

Ural Department of Russian Academy of Sciences, 620990 Ekaterinburg, Russia

* Ural Federal University, 620002 Ekaterinburg, Russia

\begin{abstract}
Using the non-equilibrium statistical operator method, we have investigated the spin transport through the interface in a semiconductor/ferromagnetic insulator hybrid structure. We have analyzed the approximation of effective parameters, when each of the considered subsystems (conduction electrons, magnons, and phonons) was characterized by its effective temperature. We have constructed the macroscopic equations describing the spin-wave current caused by both the resonantly exciting spin subsystem of conduction electrons and an inhomogeneous temperature field in the ferromagnetic insulator. Also, we have derived the generalized equations describing the spin-wave current propagation in the insulator and taking into account the resonant-diffusion nature of the propagation of magnons and their relaxation processes. We have shown that the spin-wave current excitation under combined resonance conditions bears a resonant nature.
\end{abstract}

